
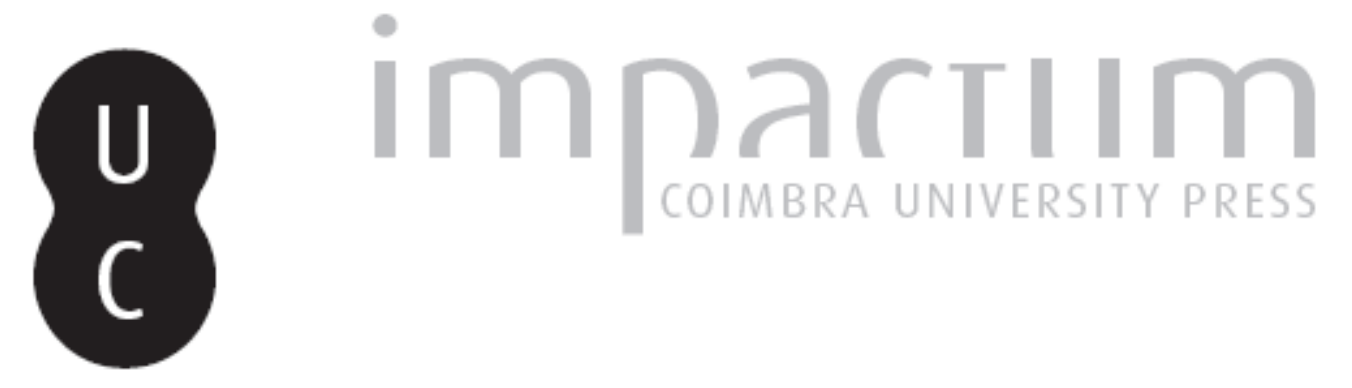

\title{
La contaminacion atmosferica como factor de riesgo: el caso de Madrid
}

\section{Autor(es): $\quad$ Fernández García, Felipe}

Publicado por: Associação Portuguesa de Riscos, Prevenção e Segurança

URL persistente:

URI:http://hdl.handle.net/10316.2/40162

DOI:

DOl:https://doi.org/10.14195/1647-7723_3_3

Accessed : $\quad$ 26-Apr-2023 02:27:50

A navegação consulta e descarregamento dos títulos inseridos nas Bibliotecas Digitais UC Digitalis, UC Pombalina e UC Impactum, pressupõem a aceitação plena e sem reservas dos Termos e Condições de Uso destas Bibliotecas Digitais, disponíveis em https://digitalis.uc.pt/pt-pt/termos.

Conforme exposto nos referidos Termos e Condições de Uso, o descarregamento de títulos de acesso restrito requer uma licença válida de autorização devendo o utilizador aceder ao(s) documento(s) a partir de um endereço de IP da instituição detentora da supramencionada licença.

Ao utilizador é apenas permitido o descarregamento para uso pessoal, pelo que o emprego do(s) título(s) descarregado(s) para outro fim, designadamente comercial, carece de autorização do respetivo autor ou editor da obra.

Na medida em que todas as obras da UC Digitalis se encontram protegidas pelo Código do Direito de Autor e Direitos Conexos e demais legislação aplicável, toda a cópia, parcial ou total, deste documento, nos casos em que é legalmente admitida, deverá conter ou fazer-se acompanhar por este aviso.

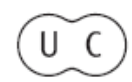




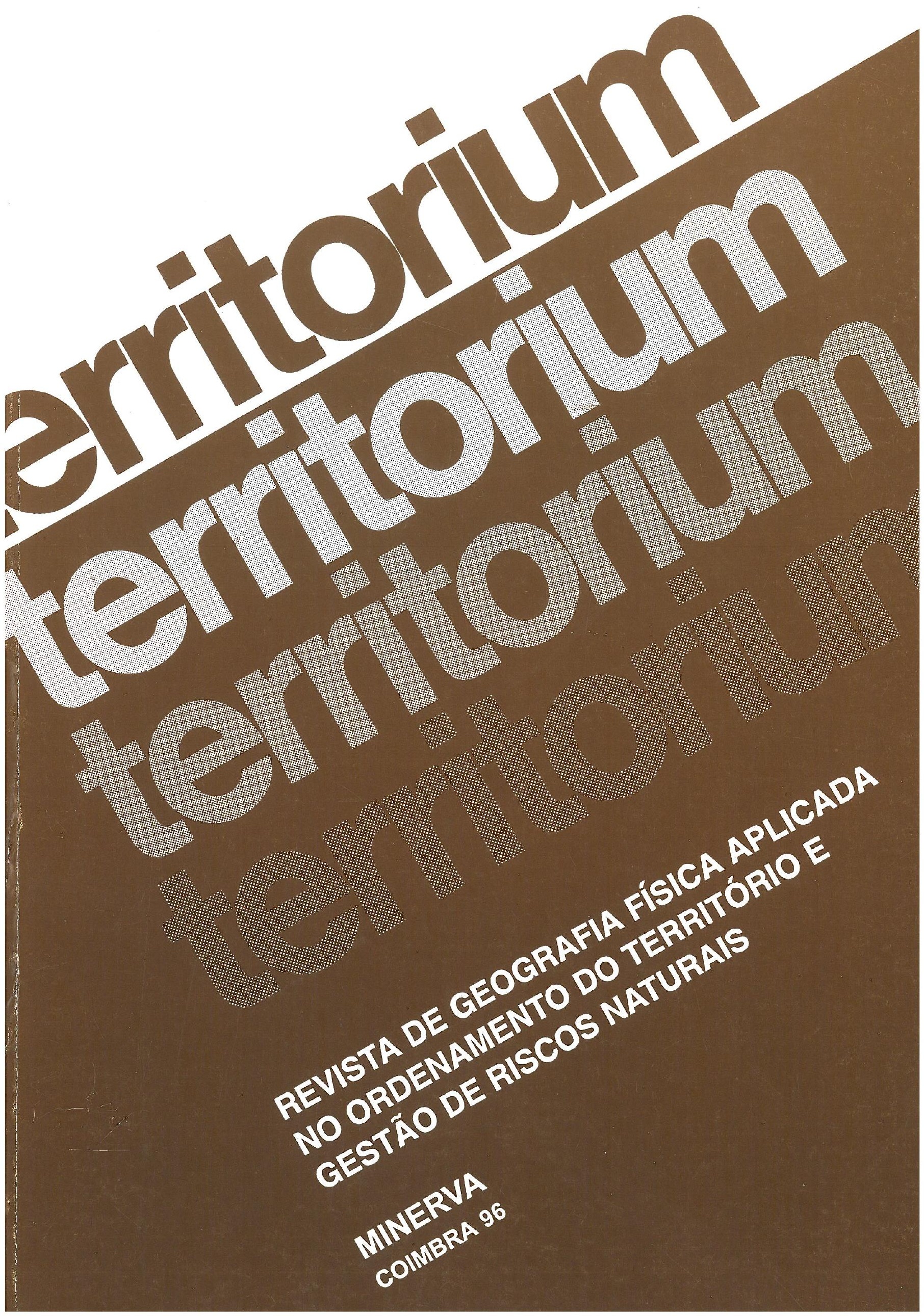




\title{
La contaminacion atmosferica como factor de riesgo: El caso de Madrid
}

\author{
Felipe Fernández García *
}

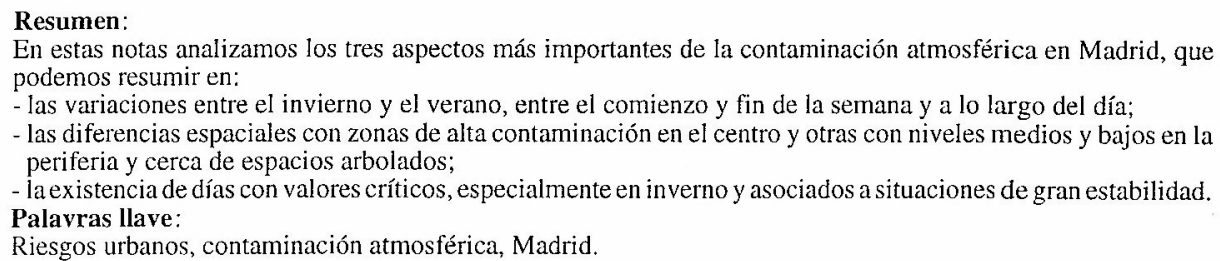

Riesgos urbanos, contaminación atmosférica, Madrid.

Resumo:

Neste artigo analisam-se os três aspectos mais importantes da poluição atmosférica em Madrid:

- variações entre inverno e verão, entre começo e fim da semana e ao longo do dia;

- diferenças espaciais, com zonas de alta poluição no centro e outras com níveis médios e baixos na periferia e junto a espacos arborizados;

- existência de dias com valores críticos, especialmente no inverno e associados a situações de grande estabilidade.

Palavras chave:

Riscos urbanos, poluição atmosférica, Madrid.

Résumé:

Dans cet article on analyse les trois aspects les plus importants de la pollution urbaine à Madrid:

- variations entre hiver et été, entre début de la semaine et "week-end" et tout au long de la journée;

- différences espacialles, avec des zones très polluées dans le centre ville et d'autres moyennement ou peu polluées en périphérie et près des espaces arborisés;

- l'existence de jours avec des valeurs critiques, notamment en hiver et associés aux situations d'une grande stabilité.

Mots clés:

Risques urbains, pollution atmosphérique, Madrid

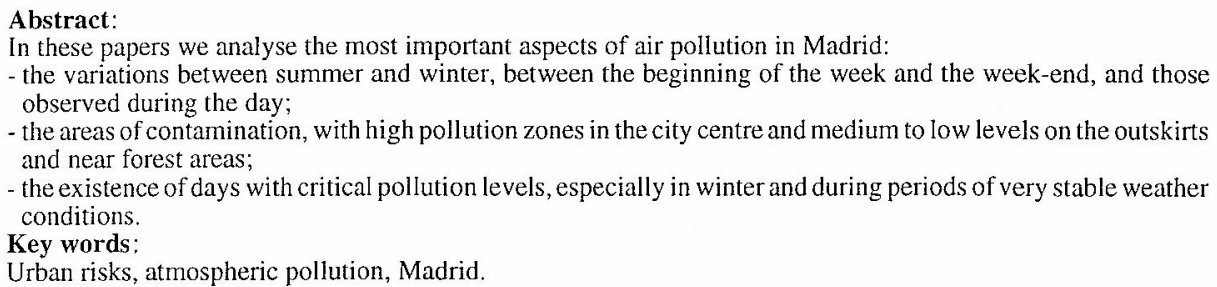

\section{I - Introducción: La contaminación atmosférica como factor de riesgo.}

La contaminación atmosférica es definida por el Engineers Joint Council como la presencia en la atmósfera de uno o varios contaminantes como polvo, humo, gases o vapores, en cantidades y duración tales que pueden incidir sobre la salud de los hombres, plantas o animales, o impedir el normal desarrollo de sus actividades. Representa, por tanto, un factor de riesgo potencial que puede afectar a las personas o a

* Departamento de Geogratia. Universidade Autónoma de Madrid. sus actividades. De hecho los episodios graves de contaminación como el del Valle del Mosa en Bélgica, a comienzos de Diciembre de 1930, provocó 60 muertos, diez veces más de la tasa de mortalidad normal; en la región de Donora, cerca del importante centro siderúrgico americano de Pittsburg, los danos fueron evaluados en 20 muertos, durante los cinco días en que duraron estas condiciones y 8500 jornadas laborales perdidas; en Londres, por último, entre el 5 y el 9 de diciembre de 1952, se registraron más de 4000 muertes, la mayor parte debidas a enfermedades respiratorias y cardiovasculares. 
En la actualidad el problema sigue vigente a tenor de los datos recogidos en el Informe del Banco Mundial sobre Desarrollo y Medio Ambiente (1992): la contaminación por partículas en las zonas urbanas es la causante de la muerte de entre 300.000 y 700.000 personas al año y del $50 \%$ de las toses infantiles crónicas.

En la tipología de riesgos naturales, sin embargo, en unos casos no se considera la contaminación como tal (ORTEGA, 1991), mientras que en otros (PITA, 1995), ésta aparece asociada al conjunto de riesgos climáticos, con un marcado carácter de riesgo inducido por la actividad humana.

En efecto, la contaminación es un hecho eminentemente antrópico, pero también climático (FERNÁNDEZ GARCÍA, 1995) y en su formación intervienen tanto los focos de emisión, como un ámbito receptor que es la atmósfera. El papel de ésta es decisivo en la concentración de contaminantes en los lugares próximos a los focos de emisión; de hecho las emisiones varían poco a lo largo del año y durante el día, pero sólo se producen episodios graves, cuando coinciden con unas situaciones atmosféricas de marcada estabilidad y escasa ventilación.

Con viento e inestabilidad los contaminantes son transportados a largas distancias, las concentraciones por unidad de volumen son menores y en las zonas próximas a los focos de emisión el factor de riesgo disminuye. No obstante, a medio o largo plazo pueden producir modificaciones en la composición global de la atmósfera, que podrían afectar al sistema climático por el reforzamiento del efecto de invernadero natural, o provocar daños en los diferentes ecosistemas naturales por el aumento de la acidez del agua de lluvia.
Desde la óptica de los riesgos climáticos asociados a la ciudad, es el primer aspecto el que nos interesa y a él dedicaremos las notas que siguen, analizando los aspectos más sobresalientes de la contaminación en Madrid.

\section{II - Características generales de la contamina- ción en Madrid}

La contaminación atmosférica en Madrid procede, fundamentalmente, de las calefacciones y el tráfico y los diversos contaminantes presentan una gran variabilidad espacial, en relación a la diversidad de tipologías urbanas y usos de suelo del conjunto urbano y una marcada estacionalidad, en la que interviene, tanto las condiciones climáticas regionales, como la periodicidad en el funcionamiento de las calefacciones y la densidad del tráfico.

Los tres aspectos que mejor caracterizan la contaminación en Madrid son: $1^{\text {(n) }}$ la variabilidad espacial; $2^{a}$ ) la variabilidad temporal, que puede ser analizada a escala horaria, diaria y estacionale y 3) la aparición de episodios críticos durante los meses de invierno, coincidiendo con situaciones anticiclónicas persistentes, en los que se superan los niveles de inmisión aceptados por la legislación vigente y que determinan un notable deterioro de las condiciones ambientales.

\section{Las variaciones espaciales de la contamina- ción en Madrid}

La red de vigilancia de la contaminación atmosférica (RVCA) se halla distribuida de tal forma que cubre practicamente la totalidad de espacios urbanos diferenciados existentes en nuestra ciudad (fig. 1).

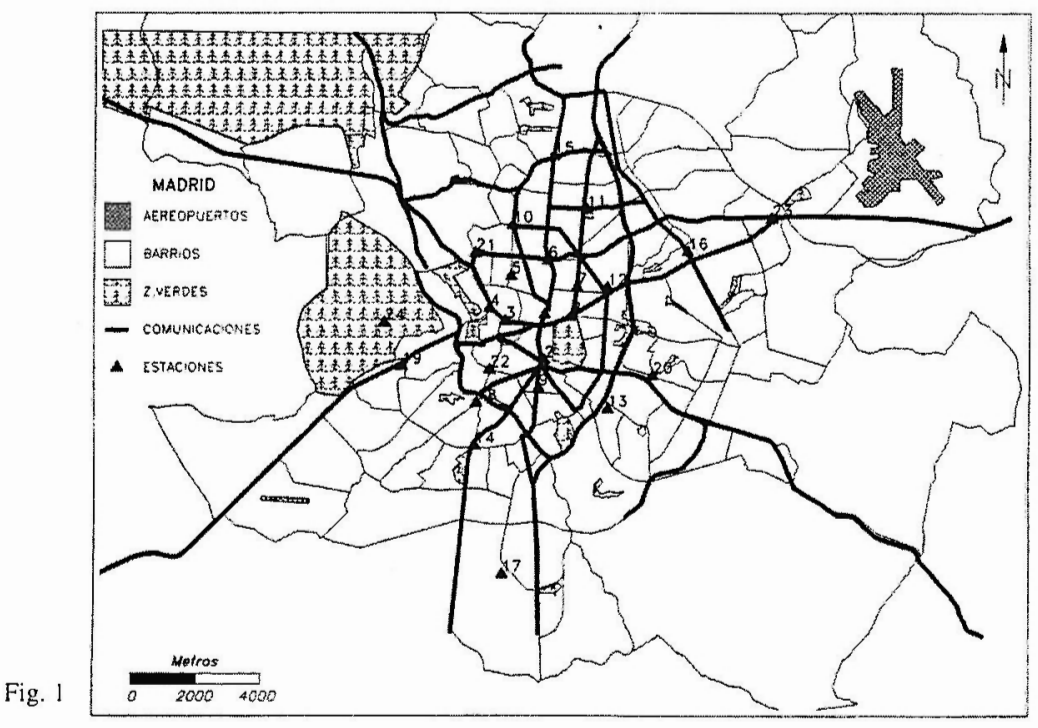


Tomando como punto de referencia el valor medio anual de $\mathrm{SO}_{2}$ y el número de días en los que se supere un determinado valor, las distintas estaciones de la red dan lugar a tres grupos bien diferenciados, que hemos denominado de elevada, media y baja contaminación.

A) Estaciones de elevada contaminación, se localizan en dos núcleos característicos: el centro urbano, con altas densidades de tráfico y edificaciones y un sector del sur de la ciudad, en el que el tráfico y las pequenas industrias son los focos principales. Los niveles de contaminación registrados en esta zona superan el valor de $60 \mu \mathrm{g} / \mathrm{m}^{3}$ para la media anual y la media diaria se mantiene por encima de $100 \mu \mathrm{g} / \mathrm{m}^{3}$ en un porcentaje de días que oscila entre el $10 \%$ y el $20 \%$ del total anual.

A.1. - El centro urbano, coincide con la zona de Protección especial, fijada en 1975 por las autoridades competentes y las estaciones donde se registran los valores más elevados de contaminación son Quevedo (5), Maranón (6), Plaza de Salamanca (7) y Cuatro Caminos $(10) .^{(1)}$

A.2. - Al sur de Madrid se localiza un punto de elevada contaminación correspondiente a la estación de Marqués de Vadillo (18), representativa de una área de trazado viario denso y estrecho, así como a la existencia de numerosas industrias medias y pequeñas.

B) Estaciones de contaminación media - En el límite señalado anteriormente como zona protegida o dentro del mismo, pero en emplazamientos muy concretos y diferentes de las características urbanas del conjunto, aparece otro grupo de estaciones en los que los niveles de inmisión medidos son menores. La media anual se mantiene entre los límites aceptables de 40-60 y el número de días que supera la concentración de $100 \mu \mathrm{g} / \mathrm{m}^{3}$ oscila entre el $10 \%$ y el $5 \%$, siendo muy escasos los que sobrepasan los $150 \mu \mathrm{g} / \mathrm{m}^{3}$.

Dentro de este grupo podemos diferenciar dos tipos:

B.1.- El primeroestá representado por las estaciones de Plaza de España (4), Plaza de Roma (12), Escuelas Aguirre (8) y Ramón y Cajal (11), cuya característica común es su emplazamiento en las proximidades de espacios verdes: Ramón y Cajal y Escuelas Aguirre en las cercanías del Parque de Berlín y del Retiro, respectivamente; las dos restantes en el interior de las plazas arboladas de donde toman el nombre.

(1)Los números entre paréntesis indican la identificación de la estación en el mapa 1.
B.2. - El otro grupo lo forman las estaciones de Recoletos (1), Luca de Tena (9), Puerta de Toledo (22) y la cercana Marqués de Vadillo (17). Salvo la primera, situada en el Paseo de la Castellana muy cerca de la zona más contaminada de Madrid, las otras dos ocupan emplazamientos más alejados, pero con tráfico intenso, aunque menor que el registrado en Castellana. En conjunto, este grupo aparece poco definido y lo podemos denominar como representativo de un área de transición.

C) Estaciones de menor contaminación, presentan claras connotaciones favorables a este rasgo distintitivo como es la posición periférica, en barrios de no muy elevada densidad urbana con amplias avenidas y espacios libres, como es el caso de Arturo Soria (16) y Moratalaz (20). La estación de Fernández Ladreda (14) se encuentra emplazada en la denominada Plaza Elíptica cerca del parque Emperatriz $\mathrm{M}^{\mathrm{a}}$ de Austria. Es esta una zona de salida de la ciudad hacia el sur, por tanto con tráfico intenso, pero que es canalizado, en gran parte, por el paso subterráneo construido bajo la Plaza, lo que podría ser un factor adicional en la explicación de los bajos niveles registrados.

Caso similar podemos aplicar a la estación número 2, Atocha, situada en las proximidades del Jardín Botánico al comienzo del Paseo del Prado, que ha experimentado un descenso significativo en los niveles de contaminación de $\mathrm{SO}_{2}$ especialmente en los últimos años, loque podría estar relacionado con la canalización de gran parte del intenso tráfico de la Glorieta a través de un paso subterráneoconstruido recientemente.

En todas ellas la media anual se sitúa por debajo de 40 y son muy raros los días en los que las concentraciones medias alcanzan los 100.

La densidad de edificaciones y el tráfico son las variables que de manera más acusada condicionan la clasificación que acabamos de exponer y en relación a ellas se puede establecer la contaminación media que se registra en las diferentes áreas de la ciudad. En la figura 2 aparecen los valores correspondientes al percentil 98 de $\mathrm{SO}_{2}$ en los diferentes barrios madrileños. El mapa ha sido elaborado a partir de la función de distribución obtenida por regresión múltiple entre los valores medios de tráfico y densidad de población correspondientes a cada uno de los barrios de Madrid y los datos de contaminación por $\mathrm{SO}_{2}$, obtenidos a partir de las superficies de tendencia calculadas por el procedimiento de la distancia media ponderada del módulo INTERPOL del Sistema de Información Geográfico IDRISI (F. FERNÁNDEZ et alt. 1993). Como se podrá observar por la localización y el 
número de estaciones de vigilancia de la contaminación representadas, en los cálculos previos se han utilizado, además de las propiamente urbanas, otras situadas en zonas del extrarradio. Los estadísticos más significativos aparecen recogidos en el Cuadro 1.

Cuadro 1

Modelo de distribución espacial de $\mathrm{SO}_{2}$ en Madrid

\begin{tabular}{|crlr|}
\hline Media diaria de $\mathrm{SO}_{2}$ & & \\
Constante & -15.90 & Fratio & 24.90 \\
Tráfico & 0.82 & $\mathrm{R}^{2}$ & 0.68 \\
Den. Pobl. & 0.52 & & \\
Percentil 50 & & & \\
Constante & -12.80 & Fratio & 29.20 \\
Tráfico & 0.74 & $\mathrm{R}^{2}$ & 0.70 \\
Den. Pobl. & 0.19 & & \\
Percentil 98 & & & \\
Constante & -21.26 & Fratio & 15.77 \\
Tráfico & 2.70 & $\mathrm{R}^{2}$ & 0.56 \\
Den. Pobl. & 0.20 & & \\
\hline
\end{tabular}

Los resultados expresados en el mapa corroboran lo anunciado anteriormente al analizar cada una de las estaciones de medición, al tiempo que permiten un análisis más preciso en cuanto a la extensión espacial de la contaminación y la influencia de los distintos factores que contribuyen a ello. Así se observan diferencias significativas entre los barrios centrales con valores superiores a $150 \mu \mathrm{g} / \mathrm{m}^{3}$ y las zonas del extrarradio con valores inferiores a $40 \mu \mathrm{g} / \mathrm{m}^{3}$.

Dentro de la ciudad las dos áreas más contaminadas se localizan a lo largo de los dos grandes ejes de tráfico que son la Castellana, que atraviesa Madrid de $\mathrm{N}$ a $\mathrm{S}$ y la $\mathrm{M}-30$, la primera vía de circulación rápida, en la actualidad dentro del área plenamente urbana. Las otras áreas urbanas con mayor contaminación corresponden a barrios con gran densidad de edificaciones, caso de Cuatro Caminos y Ríos Rosas, o con densidad urbana e industria como sería el caso de algunos barrios de la periferia sur.

Por su parte las zonas próximas a Arturo Soria y Moratalaz, periferia E. SE, aparecen con valores similares a los del extrarradio. Lo mismo ocurre hacia el $\mathrm{E}$ donde se localizan grandes extensiones boscosas de la Casa de Campo (4) y Monte del Pardo (5), que reducen considerablemente los niveles de inmisión.

Es preciso insistir en la importancia del tráfico rodado como factor de contaminación, dado que el mapa se observa de forma muy clara como los grandes ejes viarios de salida de la ciudad extienden el área de contaminación hacia el exterior. Tal ocurre hacia el este siguiendo el trazado de la autopista de Barcelona (1); hacia el SE por la de Valencia (2) y hacia el S por la de Toledo (3).

\section{EI ritmo diario y estacional}

En este apartado se pueden diferenciar, por un lado, las variaciones relacionadas con la acción conjunta de las situaciones meteorológicas y la intensidad de las actividades urbanas y, por otro, las relacionadas sólo con las actividades urbanas, especialmente el tráfico. En el primer grupo estarían las variaciones de contaminación entre el verano y el invierno, es decir las de carácter estacional; al segundo las horarias y semanales.

\subsection{Variaciones estacionales}

La época más favorable para la elevada contaminación de $\mathrm{SO}_{2}$ y partículas coincide con el semestre frío, especialmente los meses de Diciembre, Enero y Febrero (fig. 3). En ellos, además de estar en funcionamiento todos los focos de emisión (calefaccio-

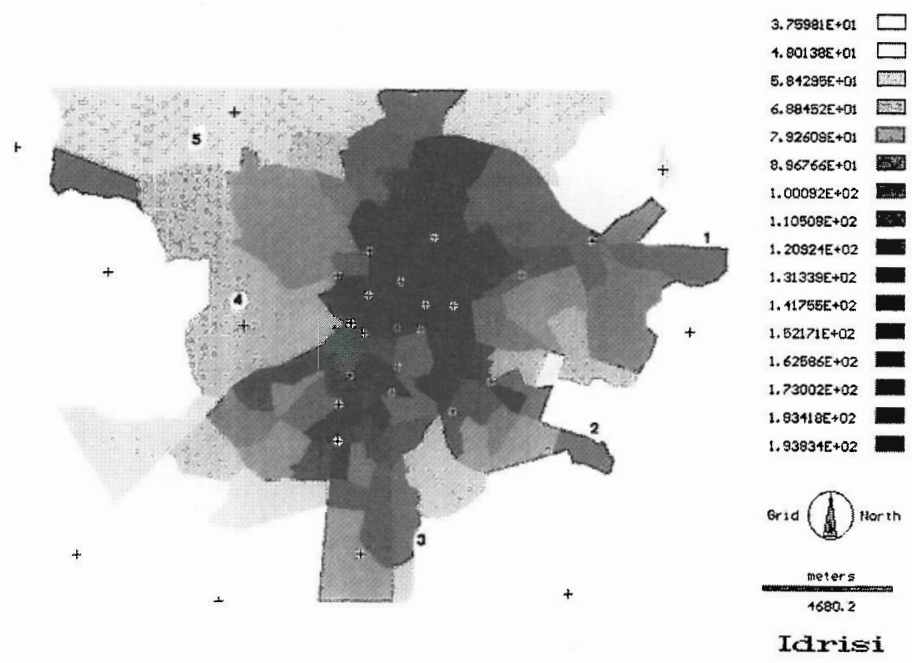


nes y tráfico), es cuando las situaciones anticiclónicas alcanzan la máxima frecuencia, con un número muy elevado de días con inversiones térmicas en superficie y viento en calma (F. FERNÁNDEZ 1986; F. FERNÁNDEZ y E. GALÁN, 1995).

Desde Marzo a Junio, predominan las situaciones inestables, el viento es más fuerte y el potencial de dispersión de los contaminantes mayor. A ello se une el apagado de las calefacciones a partir del 1 de abril.

En los meses de verano, sobre la Península Ibérica se instalan altas presiones en los niveles medios y altos de la atmósfera, pero en el interior se forman bajas presiones superficiales de origen térmico, que favorecen las turbulencias y la dispersión de los contaminantes. Por ello en estos meses raramente se alcanzan niveles de contaminación elevados, salvo en el ozono, cuya evolución está estrechemente relacionada con la fuerte insolación (fig. 3 ).

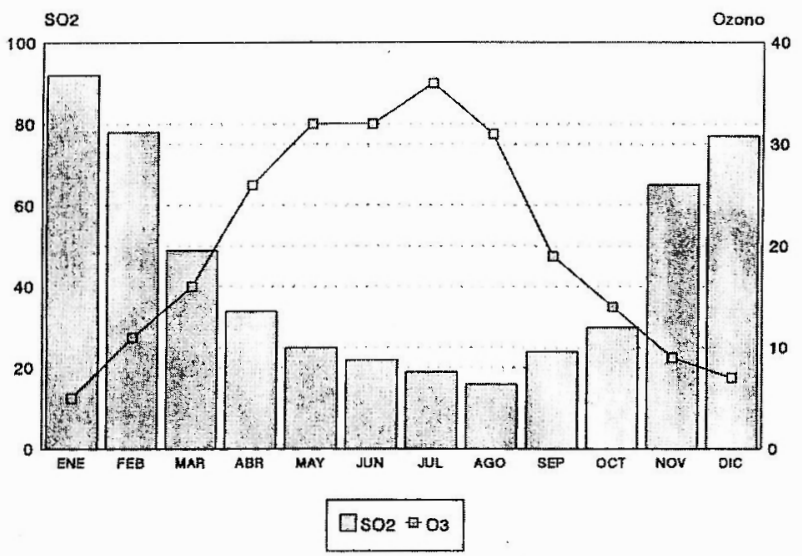

\subsection{Variaciones diarias}

La densidad de tráfico y, en menor medida, la inactividad en los grandes centros comerciales y de oficinas es la causa del ritmo semanal y horario que siguen los principales contaminantes. A lo largo de la semana, se observa un incremento progresivo desde el comienzo que culmina en un máximo el viernes; el sábado y domingo se produce un descenso significativo en la contaminación por humos y óxidos de azufre y nitrógeno (fig. 4); otros contaminantes como el monóxido de carbono $(\mathrm{CO})$ y el ozono $\left(\mathrm{O}_{3}\right)$ este descenso es menos acusado o no aparece.

Durante el día, la curva de inmisiones de $\mathrm{SO}_{2}$ sigue el ritmo de la actividad urbana, especialmente del tráfico rodado: ascenso significativo a partir de las 5 AM y máximos localizados a las 12 y las 20 horas, con un pequeno mínimo entre ambos (fig. 5).

Fig. 3 - Evolución mensual del $\mathrm{SO}_{2}$ y Ozono en Madrid Medial 9(2-93.
Fig. 4 - Evolución semanal de la contaminción en Madrid Conjunto red 1993.

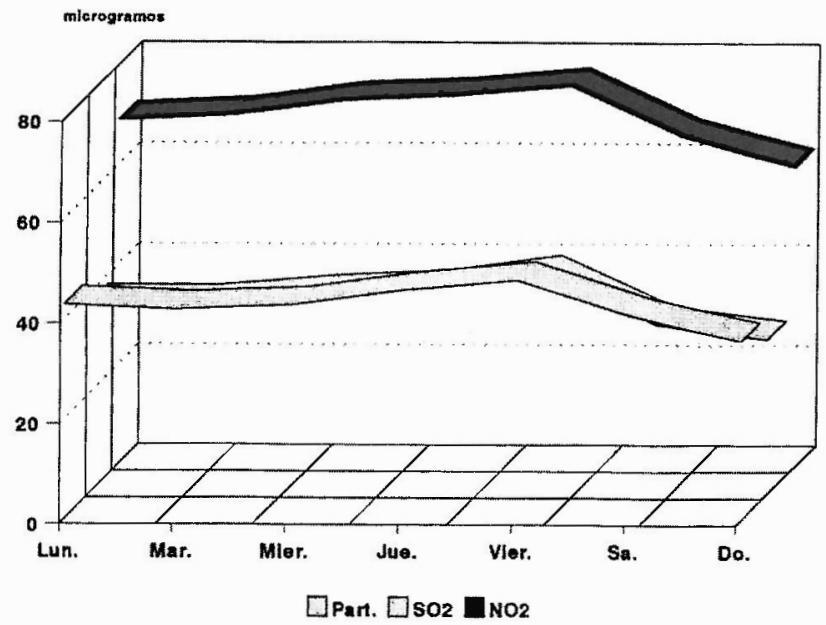




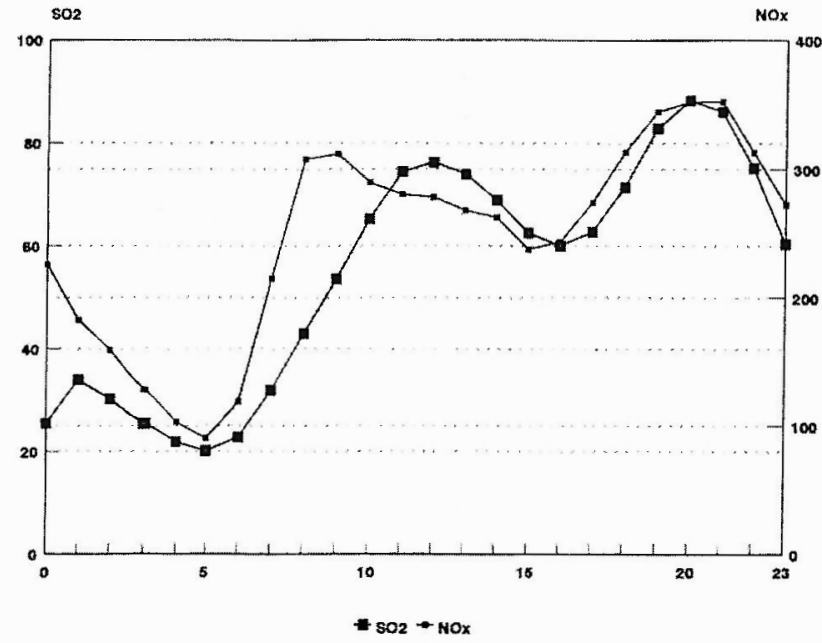

Las concentraciones de $\mathrm{NO}_{\mathrm{x}} \mathrm{y}$ otros contaminantes como los HCNM (hidrocarburos no metálicos) siguen un ritmo similar, aunque el primer máximo se adelanta respeto al $\mathrm{SO}_{2}$ (fig. 5).

El ozono, por último, sigue un ritmo diferente con un máximo principal en el centro del día, coincidiendo con la máxima insolación y el descenso del $\mathrm{NO}_{\mathrm{x}}$ y HCNM, a partir de los cuales se forma. En verano aparece otro máximo secundario de madrugada (fig. 6).
Fig. 5 - Evolución horaria de la contaminación en Madrid Conjunto do la red. Año 1993

Tomando como punto de referencia las concentraciones de $\mathrm{SO}_{2}$, los umbrales que definen los periodos críticos son el de $150 \mu \mathrm{g} / \mathrm{m} 3$, valor límite aceptado por la legislación española y el de $250 \mu \mathrm{g} / \mathrm{m}^{3}$, considerado como gravemente dañino para la salud, por tanto inaceptable, por la O.M.S. (PERSON, 1980).

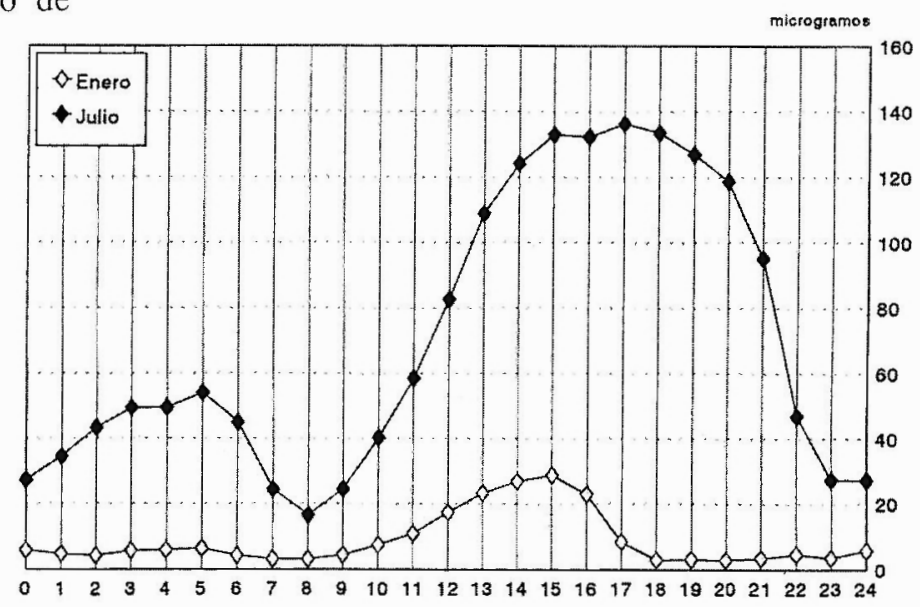

Fig. 6 - Ozono. Evolución horaria y mensual. Enero y Julio de 1993.

\section{Periodos críticos}

Definimos como tales, intervalos de tiempo de uno o varios días de duración en los que los niveles de inmisión superan los valores guía o los límites admitidos por la legislación. Normalmente coinciden con situaciones meteorológicas de gran estabilidad por lo que la época del año más favorable es el invierno y afecta de forma desigual a las diferentes áreas urbanas.

\subsection{Frecuencia}

El $80 \%$ de los días en que este contaminante presenta concentraciones mayores o iguales a $150 \mu \mathrm{g} / \mathrm{m}^{3}$ se sitúan en el invierno (49 sobre 61 en el año 1990 y 31 sobre 40 en el 91), época en la que la estabilidad, las inversiones térmicas junto al suelo y las de subsidencia, así como las calmas son más frecuentes; de hecho, al clasificar los días según las clases de estabilidad definidas por Pasquill, las 
que aparecen durante estos días son la $\mathrm{D}, \mathrm{F}$ y $\mathrm{G}$ (Cuadro 2).

En términos porcentuales, los días con altos índices de contaminación equivalen al $54 \%$ de los días invernales, el $8 \%$ del otoño y el $5 \%$ de primavera, en el año 1990; en 1991, estos porcentajes se sitúan en el $34 \%$ (invierno) y el $10 \%$ (otoño), no apareciendo en este caso ningún día en primavera. Durante el verano el riesgo de contaminación grave por $\mathrm{SO}_{2}$ es prácticamente nulo dado que predominan situaciones de inestabilidad en las capas bajas de la atmósfera y una de las fuentes principales de emisión, como las calefacciones está inactiva.
A. - La situación de enero del 90 (Cuadro 3 ) tuvo una duración de seis días y en todos los sectores de la ciudad se registraron valores elevados; no obstante éstos afectaron de forma general a todas las estaciones del centro urbano, zonas 1 y 2 , mientras que en la zona 3 únicamente lo registran Atocha (2) y Moratalaz (20). En diversos puntos de la red se alcanzaron concentraciones por encima de los $250 \mu \mathrm{g} / \mathrm{m}^{3}$ con máximos de $290 \mu \mathrm{g} / \mathrm{m}^{3}$ en la Glorieta de Cuatro Caminos (zona 1), de $288 \mu \mathrm{g} / \mathrm{m}^{3}$ en la Plaza de Luca de Tena (zona 2) y de $267 \mu \mathrm{g} / \mathrm{m}^{3}$ en Moratalaz (zona 3 ).
Cuadro 2

Frecuencia de situaciones meteorológicas (días con niveles de $\mathrm{SO}_{2} \geq 1.50 \mu \mathrm{g} / \mathrm{m}^{3}$ )

\begin{tabular}{|c|c|c|c|c|c|c|c|c|}
\hline \multicolumn{9}{|c|}{ Año 1990} \\
\hline \multirow[t]{2}{*}{ Situación } & \multicolumn{2}{|c|}{ Invierno } & \multicolumn{2}{|c|}{ Primavera } & \multicolumn{2}{|c|}{ Otoño } & \multicolumn{2}{|c|}{ Año } \\
\hline & $\mathrm{n}^{0}$ días & $\%$ & $\mathrm{n}^{\circ}$ días & $\%$ & $n^{\circ}$ días & $\%$ & $\mathrm{n}^{\circ}$ días & $\%$ \\
\hline G & 13 & 26,5 & & & 2 & 28,6 & 1.5 & 24,6 \\
\hline F & 24 & 49,0 & 1 & 20,0 & 4 & 57,1 & 29 & 47,5 \\
\hline \multirow[t]{2}{*}{ D } & 12 & 24,5 & 4 & 80,0 & 1 & 14,3 & 17 & 27.9 \\
\hline & 49 & 100,0 & 5 & 100,0 & 7 & 100,0 & 61 & 100,0 \\
\hline \multicolumn{9}{|c|}{ Año 1991} \\
\hline & \multicolumn{2}{|c|}{ Invierno } & \multicolumn{2}{|c|}{ Primavera } & \multicolumn{2}{|c|}{ Otoño } & \multicolumn{2}{|c|}{ Año } \\
\hline & $n^{\circ}$ días & $\%$ & $\mathrm{n}^{\circ}$ días & \% & $n^{0}$ días & $\%$ & $n^{\circ}$ días & $\%$ \\
\hline $\mathrm{G}$ & 14 & 45,1 & & & 3 & $3,3,3$ & 17 & 42,5 \\
\hline F & 11 & 35.5 & & & 5 & 55,6 & 16 & 40,0 \\
\hline $\mathrm{D}$ & 6 & 19,4 & & & 1 & 11.1 & 7 & 17,5 \\
\hline & 31 & 100,0 & & & 9 & 100,0 & 40 & 100,0 \\
\hline
\end{tabular}

\subsection{Rasgos caracterísiticos}

Tres son lo rasgos más significativos de estos periodos críticos: la distinta duración, la tendencia a concentrarse en invierno y el impacto desigual en las diferentes áreas urbanas.

A título de ejemplo analizamos a continuación tres secuencias de contaminación grave en Madrid: la primera secuencia tuvo lugar del 18 al 23 de enero de 1990, la segunda del 15 al 17 de febrero del mismo año y la tercera del 17 al 28 de diciembre de 1991.

Las características más importantes de cada una de ellas aparecen resumidas en los Cuadros 3,4 y 5 , donde se especifica el nivel máximo de $\mathrm{SO}_{2}$ registrado en cada una de las zonas de contaminación, valor del gradiente térmico vertical y la situación meteorológica.
B. - En el episodio correspondiente a los dias 15, 16 y 17 de febrero de 1990 (Cuadro 4) los índices de contaminación fueron muy similares al anterior, pero a diferencia de aquel la duración fue menor y únicamente afectó a varias estaciones de las zonas 1 y 2.

C. - La tercera situación analizada (del 17 al 28 de diciembre 1991) fue sin duda la más grave, tanto por su persistencia, como por el hecho de presentar los más elevados niveles de inmisión (cuadro 5). En efecto, las condiciones anticiclónicas se prolongan durante doce días consecutivos y la marcada estabilidad atmosférica provoca en algunos puntos inmisiones que rebasan los $300 \mu \mathrm{g} / \mathrm{m}^{3}$ (estación 6). Desde el punto de vista espacial vuelve a ser el centro de la ciudad el más afectado de tal forma que el conjunto de estaciones ubicadas en este sector mantienen 
territorium 3.1996

todos los días elevados niveles de contaminación ( $>150 \mu \mathrm{g} / \mathrm{m} 3$ ) frente a lo que ocurre en aquéllas que ocupan una posición más periférica (zona 3).
Para otros contaminantes como el ozono el concepto de periodo crítico es diferente a los que acabamos de analizar. Las concentraciones medias superiores a

\begin{tabular}{|c|c|c|c|c|c|}
\hline \multirow[t]{2}{*}{ Día } & \multicolumn{3}{|c|}{$\begin{array}{c}\mathrm{SO}_{2}\left(\mu \mathrm{g} / \mathrm{m}^{3}\right) \\
\text { Valor miximo registrado en la \%oma }\end{array}$} & \multirow[t]{2}{*}{$\begin{array}{c}\text { Gracticnte entre } \\
\text { superlícic } \\
\text { y } 850 \mathrm{hPal}\end{array}$} & \multirow[t]{2}{*}{$\begin{array}{l}\text { Siluación } \\
\text { metcorolsgica }\end{array}$} \\
\hline & Zona 1 & Zonit 2 & Zonis 3 & & \\
\hline 18 & $\begin{array}{l}290 \\
(10)\end{array}$ & $\begin{array}{l}270 \\
\text { (11) }\end{array}$ & $\begin{array}{l}196 \\
(29)\end{array}$ & -0.21 & $\mathrm{~F}$ \\
\hline 19 & $\begin{array}{l}241 \\
(10)\end{array}$ & $\begin{array}{l}245 \\
(9)\end{array}$ & $\begin{array}{l}226 \\
(20)\end{array}$ & -0.41 & $F$ \\
\hline 20) & $\begin{array}{l}226 \\
(5)\end{array}$ & $\begin{array}{l}263 \\
(9) \\
\end{array}$ & $\begin{array}{r}236 \\
(20)\end{array}$ & -0.10 & F \\
\hline 21 & $\begin{array}{l}199 \\
(5)\end{array}$ & $\begin{array}{l}240 \\
(9)\end{array}$ & $\begin{array}{l}222 \\
(20)\end{array}$ & 0,0 & $\mathrm{G}$ \\
\hline 22 & $\begin{array}{l}283 \\
(5)\end{array}$ & $\begin{array}{l}288 \\
(9)\end{array}$ & $\begin{array}{r}267 \\
(20)\end{array}$ & 0.20 & $\mathrm{G}$ \\
\hline 23 & $\begin{array}{l}279 \\
(5) \\
\end{array}$ & $\begin{array}{l}194 \\
(9)\end{array}$ & $\begin{array}{l}185 \\
\text { (2) }\end{array}$ & 0.30 & G \\
\hline Media & 253 & 251,5 & 222 & & \\
\hline
\end{tabular}

Cuadro 3

Situación del día 18 al 23 de enero de 1990
Cuadro 4

Situación del día 15 al 17 de febrero de 1990

\begin{tabular}{|c|c|c|c|c|c|}
\hline \multirow[t]{2}{*}{ Día } & \multicolumn{3}{|c|}{$\begin{array}{c}\mathrm{SO}_{2}\left(\mu \mathrm{g} / 1 n 1^{3}\right) \\
\text { Vator miximo registrado en la zona }\end{array}$} & \multirow{2}{*}{$\begin{array}{c}\text { Gradiente enute } \\
\text { superficie } \\
\text { y } 850 \text { hPa }\end{array}$} & \multirow[t]{2}{*}{$\begin{array}{l}\text { Situición } \\
\text { meteorolage }\end{array}$} \\
\hline & Zona 1 & Zona 2 & Zona 3 & & \\
\hline 15 & $\begin{array}{l}257 \\
(18)\end{array}$ & & & 0.42 & $\mathrm{G}$ \\
\hline 16 & $\begin{array}{l}251 \\
(5)\end{array}$ & $\begin{array}{l}177 \\
\text { (11) }\end{array}$ & & $" 0.44$ & G \\
\hline 17 & $\begin{array}{c}283 \\
(5)\end{array}$ & $\begin{array}{l}185 \\
\text { (11) }\end{array}$ & & 0.0 & $\mathrm{G}$ \\
\hline
\end{tabular}

\begin{tabular}{|c|c|c|c|c|c|}
\hline \multirow[t]{2}{*}{ Dia } & \multicolumn{3}{|c|}{$\begin{array}{l}\mathrm{SO}_{2}\left(\mu \mathrm{g} / \mathrm{m}^{3}\right) \\
\text { Valor míximo registrado en lia \%onat }\end{array}$} & \multirow[t]{2}{*}{$\begin{array}{c}\text { Gradiente contre } \\
\text { superlicie } \\
\text { y } 859 \mathrm{hPa} \\
\end{array}$} & \multirow[t]{2}{*}{$\begin{array}{l}\text { Situación } \\
\text { meteorológic: }\end{array}$} \\
\hline & Zona 1 & Zona 2 & Zona 3 & & \\
\hline 17 & $\begin{array}{l}257 \\
(10) \\
\end{array}$ & $\begin{array}{l}156 \\
(11) \\
\end{array}$ & & 0.20 & $\mathrm{G}$ \\
\hline 18 & $\begin{array}{l}323 \\
\text { (6) } \\
\end{array}$ & $\begin{array}{l}183 \\
(11) \\
\end{array}$ & & 0.19 & G \\
\hline 19 & $\begin{array}{l}234 \\
\text { (6) }\end{array}$ & $\begin{array}{l}154 \\
\text { (11) }\end{array}$ & & 0.79 & $\mathbf{G}$ \\
\hline 20 & $\begin{array}{l}194 \\
(6)\end{array}$ & & & .0 .49 & $F$ \\
\hline 21 & $\begin{array}{l}226 \\
(6)\end{array}$ & $\begin{array}{l}170 \\
\text { (11) } \\
\end{array}$ & & 0.29 & $G$ \\
\hline 22 & $\begin{array}{l}307 \\
(6) \\
\end{array}$ & $\begin{array}{l}201 \\
(11) \\
\end{array}$ & & 0.19 & $G$ \\
\hline 23 & $\begin{array}{l}261 \\
(10)\end{array}$ & $\begin{array}{l}335 \\
(9)\end{array}$ & $\begin{array}{l}290 \\
\text { (2) }\end{array}$ & 0.29 & G \\
\hline 24 & $\begin{array}{r}193 \\
(10)\end{array}$ & $\begin{array}{l}197 \\
(8) \\
\end{array}$ & & 0.39 & $G$ \\
\hline 25 & $\begin{array}{r}157 \\
(18) \\
\end{array}$ & & & -0.10 & $F$ \\
\hline 26 & $\begin{array}{c}268 \\
\text { (7) }\end{array}$ & $\begin{array}{l}186 \\
\text { (11) }\end{array}$ & & 0.49 & G \\
\hline 27 & $\begin{array}{l}275 \\
(6) \\
\end{array}$ & $\begin{array}{l}267 \\
(1) \\
\end{array}$ & $\begin{array}{l}171 \\
(2) \\
\end{array}$ & 0.30 & G \\
\hline 28 & $\begin{array}{l}301 \\
(10)\end{array}$ & $\begin{array}{l}207 \\
\text { (1) }\end{array}$ & & 0.10 & G \\
\hline Meditt & 250 & & & & \\
\hline
\end{tabular}

Cuadro 5

Situación del día 17 al 28 de diciembre de 1991 
los niveles considerados como peligrosos para la salud aparecen durante siete meses. Los umbrales que los definen son las medias en ocho horas superiores a $120 \mu \mathrm{g} / \mathrm{m}^{3}$ y valores horarios superiores a $110 \mu \mathrm{g} / \mathrm{m}^{3}$.

En el gráfico (fig. 7) se observa como el número de días con máximos en ocho horas superiores a $120 \mu \mathrm{g} / \mathrm{m}^{3}$ superan la mitad de los días del mes en mayo, junio y julio, en este último llegan a ser de 20 días.

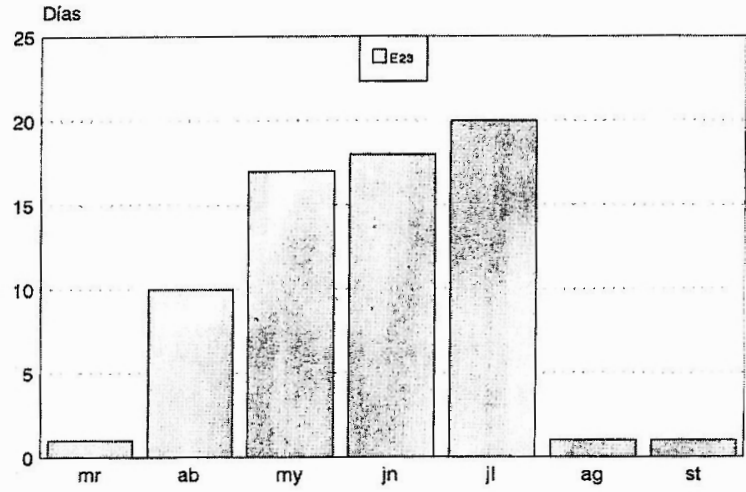

Fig. 8 - Días con máximos horarios superiores a 100 microgramos $/ \mathrm{m}^{3}$ Orono. 1943
Los máximos horarios superiores a $110 \mu \mathrm{g} / \mathrm{m}^{3}$ (fig. 8) siguen el mismo ritmo, aunque en este caso en junio y julio son más de 20 los días en que tales valores se registran. No obstante hemos de tener en cuenta que los máximos octohorarios los hemos calculado entre las 12 y las 19 , horas en las que se alcanzan los mayores niveles de concentración de ozono; el cómputo oficial considera tres periodos partiendo de las $00 \mathrm{~h}$ por 10 que las medias son inferiores al incluir horas de máximas con otras de mínima concentración.

Fig. 7 - Días con máximos en ocho horas superiores a $120 \mathrm{microgramos} / \mathrm{m}^{3}$ Orono. 199.3

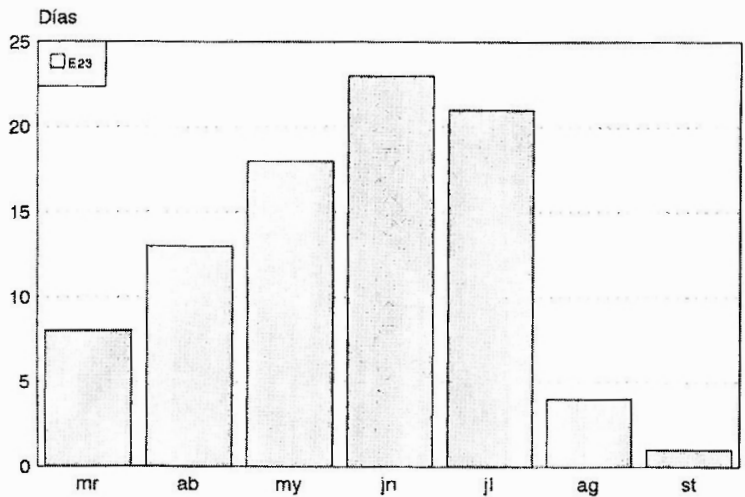


territorium 3.1996

\section{BIBLIOGRAFIA CITADA}

Ayuntamiento de Madrid (1982) - Plan de saneamiento atmosférico. Red automática de control. Madrid, Dto. de Medio Ambiente, Doc. $\mathrm{n}^{\circ} 5$

CATALÁ, J.(1976) - "Contaminación atmosférica". Madrid, Revista de la Universidad Complutense, XXV, pp. 63-93

DIAMANT, P. (1975) - Prevención de la contaminación. Madrid, Mapfre, $289 \mathrm{pp}$.

FERNÁNDEZ GARCÍA, F, GALÁN y Ga HERNÁNDEZ, E. (1993) - "Avance de un modelo de distribución espacial de la contaminación en Madrid", XIII Congreso Nacional de Geografia, Sevilla.

FERNÁNDEZ GARCÍA, F. y GALÁN, E. (1993) - "La contaminación en Madrìd: distribución espacial". $V$ Congreso de Geoquimica de España. Madrid, pp. 127-133.
FERNÁNDEZ GARCÍA, F. (1986) - El clina de la Meseta Meridional. Los tipos de Tiempo . Madrid, Ed. Universidad Autónoma, $\cdot 212 \mathrm{pp}$.

FERNÁNDEZ GARCÍA, F. y GALÁN, E. (1995) - "Episodios de elevada contaminación en Madrid" en Situaciones de Riesgo Climático en España, Creus edt. Jaca, Instituto Pirenáico de Ecología.

PERSON, G. A. (1980) - "Criterios y pautas de calidad del aire en relación con ciertos contaminantes del medio urbano" en Manual de Catidad del aire en medio urbano. Mejico, Organización Panamericana para la Salud, pp. 35-54.

PLEITE SÁNCHEZ, J.et ait. (1990) - Guia para la evaluación de los contaminantes del aire por Dióxido de azufre y partículas en suspensión. Madrid, M. Sanidad, 117 pp.

VESILIND, A. (1975) - Environmentalpollution and control. Michigan, ed. Ann Arbor Science, 231 pp. 